Published: December 20, 2018

Corresponding Author:

James T. Arnone

Assistant Professor of Biology

William Paterson University of New Jersey

300 Pompton Rd. Wayne, NJ, USA

Tel. 973-720-3457

Email: arnonej@wpunj.edu

* All authors contributed equally to this work
REVIEW ARTICLE

\section{Recent Developments on the Role of Spatial Positioning in Gene Expression and Disease}

\author{
Ahmad Abu Hardan*, Juan M. Botero*, and James T. Arnone
}

\section{Authors' affiliations:}

Department of Biology, William Paterson University of New Jersey

\title{
Abstract
}

There are myriad mechanisms that collaborate for the proper regulation of gene expression, including the spatial positioning of genes along the chromosome. Numerous studies in many model systems have characterized the effects of genomic context on transcriptional regulation, with several recent studies expanding our understanding of the nature and mechanisms underlying this phenomenon. This mini-review will focus on these recent developments to our understanding of the role of spatial positioning in

Copyright: transcriptional regulation and how these can lead to disease.

(C) 2018 Science Publishing Group

This open access article is distributed under the terms of the Creative Commons Attribution NonCommercial License. 


\section{Spatial Positioning in Gene Expression and Disease}

\section{Background and Introduction}

Proper transcriptional regulation utilizes a multifaceted approach to ensure cellular and organismal survival. This can prompt a cellular response triggering comprehensive changes to the whole transcriptome - such as the establishment of cellular identity during differentiation and during induction of the stress response - or to fine tune expression of individual genes as necessitated by cellular demand [1]. Regardless of the size and the scope of the transcriptional changes, many mechanistic themes work in concert to maintain proper transcriptional levels within the cell. At the heart of this process is the Central Dogma of molecular biology - the production of a transient RNA intermediate that is ultimately used for synthesis of proteins by the ribosome [2].

The RNA intermediate is synthesized by a RNA polymerase that is recruited to the template strand of DNA via a combination of cis regulatory DNA sequences and trans regulatory proteins. The regulatory sequences proximal to a coding region necessary for transcription are called promoters, and the more distal regions are called enhancers. Extensive efforts have identified, documented, and integrated the regulatory DNA sequences both in humans (the ENCODE Project) and model systems (the Mouse ENCODE Consortium) to completely map these sequences. There are many more regulatory sequences than protein coding regions, with tens of thousands more promoter and hundreds of thousands more enhancer sequences than transcribed coding regions $[3,4]$. The proteins that directly bind to these regulatory regions are called transcription factors (TFs). TFs are DNA binding proteins that bind in a sequence-specific manner, discriminating between their appropriate binding site and many closely related, but not identical, consensus sequences [5]. These transcriptional regulators work in a combinatorial fashion to allow for diverse regulatory effects to modulate the transcription of corresponding target loci [6]. TFs can function in singular, or they can interact with each other in complexes, allowing for passive TF binding, collaborative TF binding (e.g. TF dimerizing), or TFs forming larger enhanceosomes prior to target locus binding [7]. These proteins and their associated complexes have the ability to discriminate bona fide targets with high specificity.

The DNA binding proteins will frequently interact with additional protein complexes, such as the Mediator complex, which integrates these complex binding combinations and facilitates direct communication with the RNA polymerase [8]. These interactions often trigger subnuclear rearrangements, causing the chromosomes to bend and loop, allowing these elements to exert their regulatory effects from a distance [9]. Additional layers of transcriptional regulation include chromatin management, with changes to both the positions of histones and the covalent modification to the histone 
tails- establishing an active (or an inactive) transcriptional state [10]. The subnuclear arrangement of the chromosomes and their three-dimensional configuration within the nucleoplasm, plays a significant role in transcriptional regulation with the formation of chromosomal territories $[11,12,13]$.

It is recognized that transcription is influenced by genomic layout - the two-dimensional distribution of genes along the chromosomes. The spatial arrangement of certain loci is essential for proper regulation during development, such as the formation of higher order structures that allow for the beta-globin genes to interact with their locus control region [14]. Some regulatory elements have the ability to initiate transcription on both strands of DNA, allowing for the co-regulation of certain gene pairs, such as the histone protein coding genes which are found in a divergent $(\leftarrow \rightarrow)$ orientation [15]. While both of these examples attest to the importance of the spatial positioning of genes along the chromosome for proper gene regulation, recent developments suggest that the distribution of genes throughout the genome may play a much more significant role in transcriptional coordination than initially appreciated. Spatial positioning is especially important for the regulation of functionally related genes, those that are in the same cellular and molecular pathways and are coordinately expressed as a regulon [16]. This review will to highlight developments on the role of spatial positioning in proper transcriptional regulation and the implications for human disease.

\section{The Telomere Proximal Effect and Position Effect Variegation}

The telomere proximal effect (TPE) was initially identified in the budding yeast, Saccharomyces cerevisiae, when it was found that the insertion of URA3, TRP1, or an ADE2 reporter gene in various locations and orientations adjacent to the chromosomal telomeres led to transcriptional repression of the corresponding reporter. The TPE is distance dependent; as the region between the telomere and a reporter increased, so does expression of the reporter gene [17]. The transcriptional repression caused by the TPE is due to the assembly of transcriptionally inactive heterochromatin that forms at the telomeres and spreads outwards in a continuous fashion mediated by the silent information regulator (SIR) protein and the structural maintenance of chromosome (SMC) protein complexes $[18,19]$. The TPE is not a phenomena that is unique to $S$. cerevisiae or fungi, the effect is widely conserved throughout eukaryotes - including in humans. The longer the telomeres, the greater the range and the extent of transcriptional silencing. The mechanism underlying TPE silencing is also conserved, with silencing in humans dependent on SIRT6 and histone deacetylation [20, 21]. 
Position effect variegation (PEV) is a similar phenomenon initially identified and characterized in the fruit fly, Drosophila melanogaster, where the integration of a reporter construct adjacent to a silenced genomic locus results in gene silencing [22]. As in the case with the TPE, the PEV was identified by the silencing a reporter gene, resulting from the spread of heterochromatin in the adjacent gene into the region with the reporter. PEV is distance dependent as well, with the requirement of spatial proximity to initiate gene silencing $[22,23]$. Both phenomena illustrate how the genomic positioning of a reporter gene is influenced via changes to the local chromatin structure inherited at the site of integration.

\section{Eukaryotic Gene Clusters and Correlated Domains of Expression}

Recent advances in high throughput, genome wide technologies, discovered that many species exhibit correlated expression of genes located in close spatial proximity along the chromosome. Microarray studies allow for the characterization of the entire genome, permitting transcriptional characterization of every gene simultaneously, and these studies identified localized clusters of correlated gene expression in: the zebrafish, Danio rerio, the flowering mustard plant, Arabadopsis thalania, the fruitfly, Drosophila melanogaster, the budding yeast, Saccharomyces cerevisiae, the nematode worm, Caenorhabditis elegans, and in humans, Homo sapiens [24, 25, 26, 27, 28, 29]. These clusters of coordinated gene expression are distributed throughout the genome, typically limited in size and scope to domains of 2-10 genes. Although larger domains of correlation have been observed, this is not the norm - with the exception being regions reminiscent of operon-like organizations seen in C. elegans $[26,27,28]$.

A recent comprehensive comparative analysis revealed this correlated expression is a fundamental characteristic of transcription conserved throughout widely divergent eukaryotes [30]. There is a global positive transcriptional correlation in eukaryotes, and the distance of this effect scales to genome size: extending for $1 \mathrm{kB}$ window in the S. cerevisiae (genome size approximately 12 megabases), extending for an 8kB window in C. elegans (genome size approximately 100 megabases), and extending for $329 \mathrm{kB}$ in humans (genome size approximately 3.2 gigabases) [30]. The genomic signatures demarcating the boundaries that limit the influence of a gene upon its neighbors most likely depends on a combination of chromatin structures and the Mediator complex [31, 32, 33]. There is also extensive variation seen at the level of individual loci, however, consistent with previous observations that found regions of anti-correlated transcription at certain loci [34]. 


\section{Transcriptional Interference of Reporter Gene Expression in Eukaryotic Organisms}

Further evidence of the intimate relationship between gene expression and chromosomal organization can be observed during gene activation. The integration of the kanamycin reporter gene (the $K A N^{R}$ gene), the highly-expressed marker used in the yeast-deletion library, can result in the transcriptional activation of the gene directly adjacent to marker placement; this is called the 'neighboring gene effect' (NGE) [35]. The frequency of this interaction varies - with the NGE altering the expression of up to 800 genes (between $7-15 \%$ of the genome). Follow-up analysis uncovered this effect resulted in the misannotation of over 9,000 genetic interactions $[35,36]$. The NGE is not an artifact specific the $\operatorname{KAN}^{R}$ reporter construct - extensive variation in transcription is documented for the genome wide integration of the red-fluorescent protein (RFP), green-fluorescent protein (GFP), and LacZ reporters that were independent of promoter strength or integration site [37].

Those who utilize synthetic biology approaches for industrial and medical applications must understand the effect of spatial positioning in modulating expression. The induction of a gene results in the activation of the surrounding genes in humans, consistent with a model whereby this activation triggers 'ripples' - analogous to a pulse of activation that radiates outward over tens of kilobases [38]. High-throughput screens have been designed to rapidly characterize the effects of random targeted integration of reporter constructs genome-wide to characterize chromosome context on transcript levels in a reporter and promoter specific manner in eukaryotic cell lines, using 'thousands of reporters integrated in parallel' (termed TRIP). This approach demonstrates that the site of integration can vary as much as a thousand-fold at the RNA level based chromosomal context [39, 40].

All these studies clearly illustrate that the transcriptional differences initially characterized at heterochromatic loci are not simply limited to the TPE or PEV or localized to loci on a small scale. The transcriptional permissiveness of genomic loci vary greatly, resulting in significant differences to the levels of gene expression for reporter constructs observed at the level of RNA expression.

\section{Adjacent Gene co-Regulation}

Certain functionally-related gene families exhibit a non-random genomic distribution. The first documented instance was identified in S. cerevisiae for the genes involved with the biogenesis of the ribosome - both the ribosomal protein (RP) gene family and the rRNA and ribosome biosynthesis (RRB) 
gene family $[41,42,43]$. This distribution was not limited to $S$. cerevisiae, the clustering of the RP and the RRB gene families was observed in both the opportunistic pathogen, Candida albicans, and the distantly related fission yeast, Schizosaccharomyces pombe [43]. When the actual paring relationships were investigated it was found that the individual paired genes differed among species, although the absolute numbers of genes functionally clustered (e.g. the overall number of pairings that exist) remained relatively constant. Follow-up comparative analysis found a non-random genomic distribution exists in both the RP and RRB gene families, and it is wide-spread amongst eukaryotes [44].

One representative RRB gene pair was functionally dissected in order to determine the transcriptional dependency the genes have relative to their spatial arrangement. The RRB genes, MPP10MRX12, were selected for analysis due to their convergent $(\rightarrow \leftarrow)$ orientation. Only MPP10 contains the canonical promoter motifs that are characteristic of the RRB gene family, which both genes require for proper transcriptional regulation $[42,43]$. Disruption of this pairing relationship via the insertion of a reporter construct managed to uncouple the expression of this pair - although only when the insert was transcriptionally active, as a silenced reporter construct did not alter the expression of this RRB pairing [45]. This phenomena is called 'adjacent gene co-regulation' (AGcoR), which is a separate and distinct phenomena from the NGE as this represents a bona fide, in vivo requirement of spatial proximity in coordination with gene regulation of functionally related gene clusters. While this is not the first characterization of the effect that genome positioning plays in transcriptional regulation, it is the most thoroughly characterized locus.

Building on these findings, a recent study focused on performing a systematic and comprehensive analysis of the extent and the conservation of AGcoR, using budding yeast as a reference. Using the Gene Ontology consortium's designation for molecular function, approximately $25 \%$ of gene families exhibit a non-random genomic distribution. The arrangement correlates with tighter transcription for threequarters of the functional clusters compared to the unpaired members within the same family [16]. This relationship also resulted in anti-correlated expression for several clusters, consistent with previous reports of this phenomena [34].

\section{The Roles of Spatial Positioning in Disease}

Spatial positioning can also alter gene expression in disease. Translocations are aberrant genomic rearrangements resulting in the relocation of a portion of one chromosome to an abnormal chromosomal 


\section{Spatial Positioning in Gene Expression and Disease}

context. The first translocation linked to disease in humans involves the two-way translocation between chromosomes 9 and 22 forming the Philadelphia chromosome, a hallmark of chronic myeloid leukemia [46]. This translocation results in the errant expression of a receptor tyrosine kinase, $B C R-A B L$, which results in a hyperactive fusion protein leading to disease. While this locus is a hot spot for translocation, there is not a nucleotide-specific breakpoint. As a result, there are multiple $B C R-A B L$ fusion forms that can form, which lead to other cancers including: acute lymphoblastic leukemia, acute myeloid leukemia, and chronic neutrophilic leukemia [47]. While the diseases are caused by the abnormal fusion protein, it is noteworthy that after the translocation event occurs both loci are transcriptionally active, albeit under the control of a new regulatory logic than prior to the crossing over [48].

The genomic re-arrangement that occurs during a translocation does not need to result in a fusion protein to cause disease. The translocation of a locus to a new context can result in a re-wiring of the gene regulatory logic in the new location leading to disease, as seen in PEV. Diffuse large B cell lymphoma tumors contain a translocation between chromosome 1 and chromosome 2 (1p12 pairs with the centromeric region of chromosome 2, resulting in the translocation). This translocation results in the abnormal assemblage of heterochromatin characterized by alterations in histone methylation (H3K9me3 and H4K20me3) and acetylation leading to repression of GMCL1 [49]. GMCL1, in turn, is a regulator of the tumor suppressor system, MDM2-P53. Downregulation of GMCL1 expression in this tumor type is correlated with a significantly shortened survival prognosis [49].

The telomere position effect can also lead to disease. The TPE contributes to the manifestation of phenotypes seen in facioscapulohumeral muscular dystrophy (FSHD). FSHD is characterized by two genomic changes: the contraction of reduced repeat numbers of the D4Z4 elements found in the peritelomeric region of chromosome $4 \mathrm{q}$ and attrition of the corresponding telomere. The 3.3kB D4Z4 repeat is ordinarily present in up to 100 copies in unaffected individuals, but individuals with FSHD have no more than 10 repeats [50]. The disease phenotype manifests itself only after telomere attrition which occurs during aging, ameliorating the TPE keeping this region repressed as heterochromatin. At this point DUX4 (present in the terminal D4Z4 repeat) and the adjacent gene FRG2 are both upregulated, leading to the muscular atrophy and weakness characteristic of this disease [51]. In a small subset of patients with FSHD (5-10\% of the population), this results in longer distance activation of the SORBS2 gene, over four megabases away, via the formation of a loop (a phenomena that is called TPE-over long distances, or TPEOLD) [52]. On a genome-wide level, position effects on transcription have also recently been characterized by their influence on patients with the human immunodeficiency virus (HIV). The TPE alters 
the transcriptional permissiveness at the site of viral integration, which alters the efficacy of treatment in certain patient populations by masking the virus during the latent period [53].

Telomere shortening can result in the formation of ring chromosomes, a genetic disorder characterized by telomeric fusion. The shortened telomeres fuse together resulting in a circular chromosome, which causes an altered TPE. In chromosome 17 ring syndrome, this fusion results in a loss of telomeric sequences resulting in the altered expression of twelve genes in this region,

including: SIRT7, FOXK2, and NXN. As in the case with FSHD, the shorter the telomeres the more significant the symptoms of this disorder [54]. This is not unique to just chromosome 17, as the misexpression of telomeric genes is a broad characteristic of ring chromosomes that can contribute to the etiology of the disease [55].

A role for adjacent gene co-regulation may also exist in disease progression. The TF HSF1 is overactive in a number of cancer types, effectively acting as a driver for malignancy [56]. When HSF1 is overactive it increases transcription in a number of target genes, re-wiring the transcriptome and giving the cell a cancer signature transcriptional profile. These target genes are clustered together in the genome, with a number of them localized to chromosome $8 q 22.3$ and 8q24.3. Functional characterization of these HSF1 targets links to pre-mRNA processing, demonstrating the first example of functionally-clustered genes being aberrantly expressed in cancers [57].

\section{Conclusions}

As many gene expression mechanisms are conserved throughout eukaryotes, it is likely that spatial positioning plays a fundamental role in the regulation of gene expression. The clustering of functionally related genes may represent an efficient evolutionary adaptation that streamlines the genome allowing efficient transcriptional coordination of many genes that are functionally related and are required in stoichiometric levels [58].

\section{Acknowledgements}

J.T.A. would like to acknowledge support and funding for this work has come from the Center for Research in the College of Science and Health and for ART from William Paterson University. He would also like to 
thank Kasey-Dee Arnone for critical readings of the manuscript. A.A.H. and J.M.B. have received support from the Garden State - Louis Stokes Alliance for Minority Participation (GS-LSAMP).

\section{References}

1. Gasch AP, Spellman PT, Kao CM, Carmel-Harel O, Eisen MB, Storz G, Botstein D, Brown PO: Genomic expression programs in the response of yeast cells to environmental changes. Molecular biology of the cell 2000, 11(12):4241-4257.

2. Crick F: Central dogma of molecular biology. Nature 1970, 227(5258):561-563.

3. Consortium TEP: An integrated encyclopedia of DNA elements in the human genome. Nature 2012, 489(7414):57-74.

4. Stamatoyannopoulos JA, Snyder M, Hardison R, Ren B, Gingeras T, Gilbert DM, Groudine M, Bender M, Kaul R, Canfield T et al: An encyclopedia of mouse DNA elements (Mouse ENCODE). Genome biology 2012, 13(8):418.

5. Todeschini AL, Georges A, Veitia RA: Transcription factors: specific DNA binding and specific gene regulation. Trends in genetics : TIG 2014, 30(6):211-219.

6. Stampfel G, Kazmar T, Frank O, Wienerroither S, Reiter F, Stark A: Transcriptional regulators form diverse groups with context-dependent regulatory functions. Nature 2015, 528(7580):147-151.

7. Reiter F, Wienerroither S, Stark A: Combinatorial function of transcription factors and cofactors. Current opinion in genetics \& development 2017, 43:73-81.

8. Allen BL, Taatjes DJ: The Mediator complex: a central integrator of transcription. Nature reviews Molecular cell biology 2015, 16(3):155-166.

9. Levine M, Cattoglio C, Tjian R: Looping back to leap forward: transcription enters a new era. Cell 2014, 157(1):13-25.

10. Weber CM, Henikoff S: Histone variants: dynamic punctuation in transcription. Genes \& development 2014, 28(7):672-682

11. Zhao R, Bodnar MS, Spector DL: Nuclear neighborhoods and gene expression. Current opinion in genetics \& development 2009, 19(2):172-179.

12. Fraser P, Bickmore W: Nuclear organization of the genome and the potential for gene regulation. Nature 2007, 447(7143):413-417.

13. Venkatesh S, Workman JL: Histone exchange, chromatin structure and the regulation of transcription. Nature reviews Molecular cell biology 2015, 16(3):178-189. 


\section{Spatial Positioning in Gene Expression and Disease}

14. Noordermeer D, de Laat W: Joining the loops: beta-globin gene regulation. IUBMB life 2008, 60(12):824-833.

15. Ewen ME: Where the cell cycle and histones meet. Genes \& development 2000, 14(18):2265-2270.

16. Eldabagh RS, Mejia NG, Barrett RL, Monzo CR, So MK, Foley JJt, Arnone JT: Systematic Identification, Characterization, and Conservation of Adjacent-Gene Coregulation in the Budding Yeast Saccharomyces cerevisiae. mSphere 2018, 3(3).

17. Gottschling DE, Aparicio OM, Billington BL, Zakian VA: Position effect at $\mathrm{S}$. cerevisiae telomeres: reversible repression of Pol II transcription. Cell 1990, 63(4):751-762.

18. Renauld H, Aparicio OM, Zierath PD, Billington BL, Chhablani SK, Gottschling DE: Silent domains are assembled continuously from the telomere and are defined by promoter distance and strength, and by SIR3 dosage. Genes \& development 1993, 7(7a):1133-1145.

19. Moradi-Fard S, Sarthi J, Tittel-Elmer M, Lalonde M, Cusanelli E, Chartrand P, Cobb JA: Smc5/6 Is a Telomere-Associated Complex that Regulates Sir4 Binding and TPE. PLoS genetics 2016, 12(8):e1006268.

20. Baur JA, Zou Y, Shay JW, Wright WE: Telomere position effect in human cells. Science (New York, NY) 2001, 292(5524):2075-2077.

21. Tennen RI, Bua DJ, Wright WE, Chua KF: SIRT6 is required for maintenance of telomere position effect in human cells. Nature communications 2011, 2:433.

22. Wallrath LL, Elgin SC: Position effect variegation in Drosophila is associated with an altered chromatin structure. Genes \& development 1995, 9(10):1263-1277.

23. Elgin SC, Reuter G: Position-effect variegation, heterochromatin formation, and gene silencing in Drosophila. Cold Spring Harbor perspectives in biology 2013, 5(8):a017780.

24. Tsai HK, Huang PY, Kao CY, Wang D: Co-expression of neighboring genes in the zebrafish (Danio rerio) genome. International journal of molecular sciences 2009, 10(8):3658-3670.

25. Williams EJ, Bowles DJ: Coexpression of neighboring genes in the genome of Arabidopsis thaliana. Genome research 2004, 14(6):1060-1067.

26. Spellman PT, Rubin GM: Evidence for large domains of similarly expressed genes in the Drosophila genome. Journal of biology 2002, 1(1):5.

27. Cohen BA, Mitra RD, Hughes JD, Church GM: A computational analysis of whole-genome expression data reveals chromosomal domains of gene expression. Nature genetics 2000, 26(2):183-186.

28. Lercher MJ, Blumenthal T, Hurst LD: Coexpression of neighboring genes in Caenorhabditis elegans is mostly due to operons and duplicate genes. Genome research 2003, 13(2):238-243. 


\section{Spatial Positioning in Gene Expression and Disease}

29. Gierman HJ, Indemans MH, Koster J, Goetze S, Seppen J, Geerts D, van Driel R, Versteeg R: Domainwide regulation of gene expression in the human genome. Genome research 2007, 17(9):1286-1295.

30. Quintero-Cadena P, Sternberg PW: Enhancer Sharing Promotes Neighborhoods of Transcriptional Regulation Across Eukaryotes. G3 (Bethesda, Md) 2016, 6(12):4167-4174.

31. Sproul D, Gilbert N, Bickmore WA: The role of chromatin structure in regulating the expression of clustered genes. Nature reviews Genetics 2005, 6(10):775-781.

32. Dobi KC, Winston F: Analysis of transcriptional activation at a distance in Saccharomyces cerevisiae. Molecular and cellular biology 2007, 27(15):5575-5586.

33. Reavey CT, Hickman MJ, Dobi KC, Botstein D, Winston F: Analysis of Polygenic Mutants Suggests a Role for Mediator in Regulating Transcriptional Activation Distance in Saccharomyces cerevisiae. Genetics 2015, 201(2):599-612.

34. Martens JA, Wu PY, Winston F: Regulation of an intergenic transcript controls adjacent gene transcription in Saccharomyces cerevisiae. Genes \& development 2005, 19(22):2695-2704.

35. Ben-Shitrit T, Yosef N, Shemesh K, Sharan R, Ruppin E, Kupiec M: Systematic identification of gene annotation errors in the widely used yeast mutation collections. Nature methods 2012, 9(4):373-378.

36. Atias N, Kupiec M, Sharan R: Systematic identification and correction of annotation errors in the genetic interaction map of Saccharomyces cerevisiae. Nucleic acids research 2016, 44(5):e50.

37. Wu XL, Li BZ, Zhang WZ, Song K, Qi H, Dai JB, Yuan YJ: Genome-wide landscape of position effects on heterogeneous gene expression in Saccharomyces cerevisiae. Biotechnology for biofuels 2017, 10:189.

38. Ebisuya M, Yamamoto T, Nakajima M, Nishida E: Ripples from neighbouring transcription. Nature cell biology 2008, 10(9):1106-1113.

39. Akhtar W, de Jong J, Pindyurin AV, Pagie L, Meuleman W, de Ridder J, Berns A, Wessels LF, van Lohuizen M, van Steensel B: Chromatin position effects assayed by thousands of reporters integrated in parallel. Cell 2013, 154(4):914-927.

40. Akhtar W, Pindyurin AV, de Jong J, Pagie L, Ten Hoeve J, Berns A, Wessels LF, van Steensel B, van Lohuizen M: Using TRIP for genome-wide position effect analysis in cultured cells. Nature protocols 2014, 9(6):1255-1281.

41. Wade C, Shea KA, Jensen RV, McAlear MA: EBP2 is a member of the yeast RRB regulon, a transcriptionally coregulated set of genes that are required for ribosome and rRNA biosynthesis. Molecular and cellular biology 2001, 21(24):8638-8650.

42. Wade $\mathrm{CH}$, Umbarger MA, McAlear MA: The budding yeast rRNA and ribosome biosynthesis (RRB) regulon contains over 200 genes. Yeast (Chichester, England) 2006, 23(4):293-306. 


\section{Spatial Positioning in Gene Expression and Disease}

43. Arnone JT, McAlear MA: Adjacent gene pairing plays a role in the coordinated expression of ribosome biogenesis genes MPP10 and YJR003C in Saccharomyces cerevisiae. Eukaryotic cell 2011, 10(1):43-53.

44. Arnone JT, Robbins-Pianka A, Arace JR, Kass-Gergi S, McAlear MA: The adjacent positioning of coregulated gene pairs is widely conserved across eukaryotes. BMC genomics 2012, 13:546.

45. Arnone JT, Arace JR, Soorneedi AR, Citino TT, Kamitaki TL, McAlear MA: Dissecting the cis and trans elements that regulate adjacent-gene coregulation in Saccharomyces cerevisiae. Eukaryotic cell 2014, 13(6):738-748.

46. Rowley JD: Letter: A new consistent chromosomal abnormality in chronic myelogenous leukaemia identified by quinacrine fluorescence and Giemsa staining. Nature 1973, 243(5405):290-293.

47. Melo JV: The diversity of BCR-ABL fusion proteins and their relationship to leukemia phenotype. Blood $1996,88(7): 2375-2384$.

48. Melo JV, Gordon DE, Cross NC, Goldman JM: The ABL-BCR fusion gene is expressed in chronic myeloid leukemia. Blood 1993, 81(1):158-165.

49. Fournier A, McLeer-Florin A, Lefebvre C, Duley S, Barki L, Ribeyron J, Alboukadel K, Hamaidia S, Granjon A, Gressin R et al: 1q12 chromosome translocations form aberrant heterochromatic foci associated with changes in nuclear architecture and gene expression in B cell lymphoma. EMBO molecular medicine 2010, 2(5):159-171.

50. Wijmenga C, Hewitt JE, Sandkuijl LA, Clark LN, Wright TJ, Dauwerse HG, Gruter AM, Hofker MH, Moerer P, Williamson $\mathrm{R}$ et al: Chromosome $4 \mathrm{q}$ DNA rearrangements associated with facioscapulohumeral muscular dystrophy. Nature genetics 1992, 2(1):26-30.

51. Stadler G, Rahimov F, King OD, Chen JC, Robin JD, Wagner KR, Shay JW, Emerson CP, Jr., Wright WE: Telomere position effect regulates DUX4 in human facioscapulohumeral muscular dystrophy. Nature structural \& molecular biology 2013, 20(6):671-678.

52. Robin JD, Ludlow AT, Batten K, Gaillard MC, Stadler G, Magdinier F, Wright WE, Shay JW: SORBS2 transcription is activated by telomere position effect-over long distance upon telomere shortening in muscle cells from patients with facioscapulohumeral dystrophy. Genome research 2015, 25(12):17811790.

53. Chen HC, Martinez JP, Zorita E, Meyerhans A, Filion GJ: Position effects influence HIV latency reversal. Nature structural \& molecular biology 2017, 24(1):47-54.

54. Surace C, Berardinelli F, Masotti A, Roberti MC, Da Sacco L, D'Elia G, Sirleto P, Digilio MC, Cusmai R, Grotta S et al: Telomere shortening and telomere position effect in mild ring 17 syndrome. Epigenetics \& chromatin 2014, 7(1):1. 
55. Guilherme RS, Moyses-Oliveira M, Dantas AG, Meloni VA, Colovati ME, Kulikowski LD, Melaragno MI: Position effect modifying gene expression in a patient with ring chromosome 14. Journal of applied genetics 2016, 57(2):183-187.

56. Mendillo ML, Santagata S, Koeva M, Bell GW, Hu R, Tamimi RM, Fraenkel E, Ince TA, Whitesell L, Lindquist S: HSF1 drives a transcriptional program distinct from heat shock to support highly malignant human cancers. Cell 2012, 150(3):549-562.

57. Zhang $C Q$, Williams $H$, Prince TL, Ho ES: Overexpressed HSF1 cancer signature genes cluster in human chromosome 8q. Human genomics 2017, 11(1):35.

58. Arnone JT: Ribosome Biogenesis: Streamlining the Genome for the Efficient Production of this Biological Nanomolecular Machine. Nanomedicine and Nanotechnology Journal 2018, 2(1):118. 